Subject headings: Energy dissipation; Bolts; Slip; Spacecraft; Spacecraft launching; Structural analysis

Corresponding Author: Prof A. D. Crocombe

Address: School of Engineering, University of Surrey, Guildford, Surrey, GU2 7XH, UK

Tel: $+44(0) 1483689194$

Fax: +44 (0)1483306039

\title{
Energy dissipation in spacecraft structures incorporating bolted joints operating in macro-slip
}

\author{
R. Wang ${ }^{1}$, A. D. Crocombe ${ }^{1}$, G. Richardson ${ }^{2}$, C. I. Underwood ${ }^{1}$ \\ ${ }^{1}$ University of Surrey, Guildford, Surrey, GU2 7XH, UK, \\ ${ }^{2}$ Surrey Space Technology Ltd., Guildford, Surrey, GU2 7XH, UK,
}

\begin{abstract}
The energy dissipation capacity of bolted joints operating in macro-slip in a simple satellite model was investigated. A novel way of modeling the macro-slip behavior was introduced and it was shown to work well. The method was established by setting the behavior of an elasto-plastic element pair to match the experimental response of a bolted joint in macro-slip. This pair consisted of two elasto-plastic solid elements forming a unit, appropriately constrained by multipoint constraints (MPCs). Experiments carried out on real satellite joint coupons were used to define the response of the joints for use in the modelling work. The energy dissipated by the
\end{abstract}


joints was obtained by calculating the area under the force-displacement hysteresis loop in the elements through non-linear transient analyses. It was found that if some of the joints operated in the macro-slip region the energy dissipating capability of the joints could be increased significantly. The response of the satellite with these joints was investigated and it was found that vibration levels were reduced significantly.

\section{Introduction}

Surrey Satellite Technology Limited (SSTL) have developed a spacecraft composed of honeycomb panels connected by many bolted joints. The energy dissipation capacity of those joints was of interest to their design team. Former research (Crocombe et al. 2006) showed that the damping capacity for plain joints operating in the micro-slip range was quite low. The percentage of the energy dissipated in the satellite deriving from the joints was not over 5\% when the structural damping coefficient was 0.04 and the excitation acceleration was $2 \mathrm{~g}$.

Ways of increasing the energy dissipation capacity of bolted joints in the spacecraft, thus decreasing the structural response, were investigated. One method of doing this is to include a visco-elastic interlayer or gasket in the bolted joint. This has been investigated and reported elsewhere (Wang et al. 2005). The levels of energy dissipation were significantly increased. An alternative approach, allowing a selection of the bolted joints to operate in macro-slip, will be evaluated here.

Bolted joints have been widely used in industry and much research has been undertaken to investigate the behavior of bolted joints. This research has generally been undertaken by computer modeling or laboratory testing. Quite often both are carried out, using experimental 
results to validate the modeling. Normally a bolted joint in shear has two, obviously different, stages. When the shear force is small, only part of the contact surface experiences slipping and this is referred to as the micro-slip stage. When the shear force is large enough the entire contact surface slips and this is referred to as the macro-slip stage. Many models and techniques have been developed to investigate the behavior of bolted joints, for example: a) Coulomb damping

model (Hitchings 1992), b) functional model (Gaul et al. 1997), c) mass-spring-dashpot model (Esmailzadeh et al. 1996), d) Elasto Slip Model (ESM) ( Gaul and Nitsche 2001), e) Valanis Model (Gaul et al. 1995, 1997, and 2001), f) LuGre Model (Gaul and Nitsche 2001, Kess 2002), g) empirical model (Ungkurapinan et al. 2003) and a wide range of detailed finite element bolted joint models (Sun 1989, Krzyzanowski 1999, Gantes and Lemonis 2003 etc.) .

Although these bolted joint models have been developed, there is still much work to be done to incorporate them in large structures such as satellites. A detailed FE model of a bolted joint can provide a good approximation in both micro-slip and macro-slip. However, such a model also cannot be used in a global satellite model because the required level of detail would be too high.

An efficient finite modeling method was developed to investigate the effect of macro-slip in bolted joints on the response of spacecraft and is presented in detail in the following sections.

\section{Model development for a spacecraft incorporating bolted joints operating in macro-slip}

\section{The elasto-plastic material unit}

Elasto-plasticity has been used in this paper. However, it was not used to model a physical elastoplastic solid, but to represent the response of a bolted joint in macro-slip. It will be shown that 
they exhibit similar behaviour under certain conditions.

In order to investigate how an appropriately constrained solid element with an elasto-plastic material works, the single element model, shown in Figure 1, was created. An elastic modulus, a yield stress and a small hardening slope were assigned to the elasto-plastic material. A von Mises

yield function and isotropic hardening rules were used. The base of the element was fully fixed and the top was constrained by multipoint constraints (MPCs) so that all nodes experienced the same displacements. One node was assigned a cyclic displacement in the $\mathrm{x}$ direction and nonlinear static analysis was carried out. The resulting force-displacement response is shown in Figure 2. Comparing this figure with the force-displacement response of a bolted joint (Figure 3, experimental data from our former research), it can be seen that they are very similar.

It is clear that if the correct material parameters were used in the solid element model, its behavior will be equivalent to the bolted joint. The question is a) how to correlate them and b) how to then incorporate such elements into the satellite model to represent the joints. In the following sections the technique will be introduced. Some further experimental data were obtained to test the response of the actual satellite joints. These are discussed and the results are used to validate the method.

\section{Experiments on the satellite bolted joints}

It was necessary to undertake strength and stiffness tests on the satellite joints to validate the design. Some panel coupons were manufactured for this purpose. Figures 4 and 5 illustrate the coupons used. They provide two views of the panel to panel (in-plane) joint and the corner joint respectively. 
The materials used were the same as the main satellite panels, aluminium (2014) skins and aluminium honeycomb (5056) cores. The size of the coupons was $100 \times 60 \times 20 \mathrm{~mm}$. M5 titanium fasteners and washers were used. The connecting strips were quite different. The in-plane joints had aluminium (7075) connecting strips on both sides of joint and these strips contained five, $6 \mathrm{~mm}$ holes. The size of the connecting strips was $94 \times 21 \times 2 \mathrm{~mm}$. There were four pins on each strip to locate the bolts in the middle of the holes, this providing uniform clearance. A titanium nut plate, in which there were five threaded holes, was used instead of normal nuts. In the corner joint there were no connecting strips but a titanium "prism" with ten threaded holes. These configurations were designed to meet the joint functional and manufacturing requirements and were used in the real satellite.

Static tests were carried out on these joints in an Instron 5500R tensile testing machine. The test coupon was mounted in a steel fixture and shear load was exerted as shown in Figure 6. The tests were controlled by an LVDT (Linear Variable Differential Transformer), which is a sensitive displacement measuring instrument. The test speed was set to $0.002 \mathrm{~mm} / \mathrm{s}$.

Some tests were carried out on a 5 fastener coupon, others were carried out on a 3 fastener coupon (the first and the fifth fastener were removed). Pre-load bolt torques of $10 \mathrm{Nm}$ and 20 $\mathrm{Nm}$ were used. The shear load was obtained from the load cell and the relative displacement of the joint was obtained from the LVDT.

Figure 7 shows some typical test results. The micro-slip and macro-slip parts are obvious. It can be seen that for this kind of joint in macro-slip there was still some residual stiffness. 
$\underline{\text { Satellite model with bolted joints that can operate in macro-slip }}$

\section{Description of the model}

The simplified satellite model, shown in Figure 8, was created. This satellite was composed of 7 honeycomb panels which were modeled with 2412 shell elements. The behavior of the corner joint, shown in Figure 5, was investigated. It can be seen that if macro-slip occurs it will take place on the two surfaces of the connecting prism. The macro-slip on the surface perpendicular to $\mathrm{x}$ axis will be in the $\mathrm{y}-\mathrm{z}$ plane and the macro-slip on the surface perpendicular to $\mathrm{z}$ axis will be in the x-y plane. Since the response of an appropriately constrained elasto-plastic solid element has been shown to be similar to joint macro-slip, two properly constrained elasto-plastic elements can be introduced to model the macro-slip on these two planes. These two elements have been called a bolted joint unit.

The following approach was used in this work. A joint unit was composed of two connected 8 node brick elements as shown in the inset in Figure 8. Clearly, this does not model the actual geometry of the joint, however, the overall behavior of the unit is very close to the real joint. The four nodes on the top of element A were connected by MPCs so that they experienced the same displacement. The same was true for the four nodes on bottom of element A and the four nodes on the front and back of element B. The bottom of element A was connected to the top panel of the satellite and the back of element B was connected to the front panel of the satellite. These two elements (top face of element A and front face of element B) were connected by another MPC in all three directions. The top and bottom of element A were connected by 4 rigid springs, as were the front and back of element B. This ensured that the elements only deformed in in- 
plane shear. In this way, element A modeled the relative movement of joints in the plane perpendicular to $\mathrm{z}$ axis and element $\mathrm{B}$ modeled the relative movement of joints in the plane perpendicular to the $\mathrm{x}$ axis.

Such a joint unit can model a single fastener joint or a joint with more than one fastener depending on the parameters used.

In the simple satellite model in Figure 8 nine bolted joint units have been included on two of the top edges while eight bolted joint units have been included on two of the lower edges giving 34 bolted joint units in total. Each unit represented 3 specific fasteners. Due to the symmetry of the structure the bolted joint units were divided into 9 representative groups as shown in Figure 8 .

Four springs were used at the base of the satellite model. They connect the excitation points to the four satellite release points. These springs were used to model the real connection between the launch vehicle and the satellite. At the same time they have been used to calculate the energy input to the satellite.

\section{Parameter correlation}

In order to ensure that this model worked as expected, a simple model, shown in Figure 9, was created. It was the same as the bolted joint unit used on the satellite and shown in Figure 8, but was independent of the satellite. There were two coincident nodes in the middle, where the unit would normally be attached to the satellite. One was constrained in all six degrees of freedom and the other was excited at $\mathrm{z}$ direction. 
One experimental result shown in Figure 7 (Torque of 20 N.m) was used to determine whether the model in Figure 9 could be used to represent the behavior of the real joint.

A bi-linear curve was drawn to represent the test results as shown in Figure 10 and this was used to fit the model in Figure 9. To do this the relationship between $F y$ (the force at which macro-slip occurs), $K_{1}$ (the slope of the micro-slip stage), $K_{2}$ (the slope of the macro-slip stage) and the material properties of solid element must be found. The important properties for the non-linear material model being used were the shear modulus $G$, the Poisson's ratio v, the hardening slope $K$ and the yield point $\sigma_{y l d}$.

The yield point was defined by the von Mises stress:

$$
\sigma_{v m}=\frac{1}{\sqrt{2}}\left[\left(\sigma_{x}-\sigma_{y}\right)^{2}+\left(\sigma_{y}-\sigma_{z}\right)^{2}+\left(\sigma_{z}-\sigma_{x}\right)^{2}+6\left(\tau_{y z}\right)^{2}+6\left(\tau_{z x}\right)^{2}+6\left(\tau_{x y}\right)^{2}\right]^{1 / 2}
$$

where $\sigma_{x}, \sigma_{y}$ and $\sigma_{z}$ are normal stresses; $\tau_{y z}, \tau_{z x}$ and $\tau_{x y}$ are shear stresses.

Due to the manner of the constraint and the excitation of the joint unit in Figure 9, one shear stress $\tau$ will dominate and all others can be neglected. The same has been found for the bolted joint unit in the satellite model when the satellite was excited in one direction at the base. Thus the yield point is

$$
\sigma_{y l d}=\sqrt{3} \tau=\sqrt{3} \frac{F_{y}}{A}
$$

where $\mathrm{A}$ is the area of the shear surface of the bolted joint unit.

The relationship between $K 1$ and $G$ can be obtained from the simple stress and strain relationship: 


$$
K_{1}=G \frac{A}{L}
$$

where $\mathrm{L}$ is the length of the edge of the brick element perpendicular to the shear surface. That is

$$
G=K_{1} \frac{L}{A}
$$

Poisson's ratio $v$ was also required to define the elastic properties of the material. This was set to a value of 0.4

The only remaining essential material property is the hardening slope $\mathrm{K}$ of the equivalent stressplastic strain curve. According to Nastran documentation, the hardening slope was defined as

$$
K=\frac{E^{T}}{1-\frac{E^{T}}{E}}
$$

where $\mathrm{E}$ is the elastic modulus and $E^{T}$ is the slope of the uniaxial stress-strain curve in the plastic region.

From equation (4) it can be seen:

$$
G_{2}=\frac{K_{2} L}{A}
$$

where $G_{2}$ is the shear modulus of the plastic region. So

$$
E^{T}=2 G_{2}(1+v)
$$

By using equations (4)-(7), it can be shown that:

$$
K=\frac{2 K_{1} K_{2} L(1+v)}{A\left(K_{1}-K_{2}\right)}
$$

From Figure 10, it can be found: 


$$
\begin{aligned}
& K_{1}=5.549286 \times 10^{8} \mathrm{~N} / \mathrm{m} \\
& K_{2}=1.640441 \times 10^{7} \mathrm{~N} / \mathrm{m} \\
& F_{y}=7769 \mathrm{~N}
\end{aligned}
$$

In the model used, $A=0.0025 \mathrm{~m}^{2}, L=0.05 \mathrm{~m}$. Substituting these data into equations (2), (4) and (8), gives:

$$
\begin{aligned}
& \sigma_{\text {yld }}=5382521 \mathrm{~N} / \mathrm{m}^{2} \\
& G=1.109857 \times 10^{10} \mathrm{~N} / \mathrm{m}^{2} \\
& K=946630.5 \mathrm{~N} / \mathrm{m}
\end{aligned}
$$

The force and displacement of the free node in Figure 9 is shown in Figure 11. The experimental results (the idealized black bi-linear curve in Figure 10) are also shown. It can be seen the elastoplastic model represented the joint behavior very well.

\section{Effects of preload on the stiffness of the joints}

The macro-slip of a joint is controlled by the preload in the bolt. It is likely that when the preload of the joint changes the stiffness may also change. Two series of experiments were carried out on the corner joints to assess this effect. The results are shown in Figure 7 where it can be seen that the stiffness does change. Two stiffness values can be obtained from the figure. Stiffnesses for intermediate torques can be interpolated between these values at the corresponding torque.

It can be found from Figure 7 that:

$$
\begin{aligned}
& F_{y 1}=2841 \mathrm{~N} \\
& F_{y 2}=7769 \mathrm{~N}
\end{aligned}
$$




$$
\begin{aligned}
& K_{11}=1.56333 \times 10^{8} \mathrm{~N} / \mathrm{m} \\
& K_{12}=5.549286 \times 10^{8} \mathrm{~N} / \mathrm{m}
\end{aligned}
$$

where $F_{y}$ and $K_{1}$ were defined in the previous section. The second subscripts 1 and 2 represent the case of torques of $10 \mathrm{Nm}$ and $20 \mathrm{Nm}$ respectively. Assuming that the stiffness of the joint varied linearly with the macro-slip force it can be shown that:

$$
K_{1}=80883.8 F_{y}-7.34579 \times 10^{7}
$$

Substituting into equation (4) and making use of equation (2), the following modulus-yield stress relation can be found:

$$
G=46698.3 \sigma_{y l d} L-\frac{L}{A} \times 7.34579 \times 10^{7}
$$

\section{Numerical results from the simple satellite model}

\section{Energy dissipation in the satellite with joints operating in macro-slip}

A value of 0.04 was assumed for the structural damping coefficient of the satellite model. This is consistent with the approach adopted for the study of micro-slip, reported elsewhere (Crocombe et al. 2006). The excitation acceleration applied was $2 \mathrm{~g}$ in the y direction. These parameters were used in both the frequency response analyses and the transient analyses discussed in the following sections. The energy dissipation capability at different macro-slip forces was investigated.

Initially, it was assumed that the bolted joints were pre-loaded to a torque of $20 \mathrm{Nm}$. In this case all the bolted joint unit elements had $G=1.109857 \times 10^{10} \mathrm{~N} / \mathrm{m}^{2}$. The hardening slope had a 
negligible effect on the energy dissipated and was ignored in the analyses. Normal modes analysis was carried out to find the first natural frequency, which was $34.97 \mathrm{~Hz}$. A frequency response analysis was then carried out at this frequency and the von Mises stress in each joint unit was obtained. The corresponding stress in the satellite body is shown in Figure 12. It shows clearly that the joints in the middle of the edge transfer more force and the joints at the corner transfer less. It should be noted that in both normal modes analyses and frequency response analyses only the elastic properties of the joint units were used. This precludes any macro-slip.

Following these analyses the yield stress of the elements were set to $80 \%$ and $50 \%$ of their corresponding stresses, obtained from the (no macro-slip) analyses above. The elastic stiffness of the elements was also changed according to equation 10 . Due to the symmetry of the model and load in the $\mathrm{x}$ and $\mathrm{y}$ axes, only a quarter of the joints needed to be investigated in detail. Analysis of the results indicated that both elements in a given bolted joint unit experienced almost the same level of load and deformation. Thus only nine elements were considered. All the other elements had the same response as one of these nine elements. The nine elements are indicated in Figure 8. Data for these were obtained and are shown in Table 1. Due to the stiffness change the first natural frequency of the structure should decrease slightly. Normal modes analyses were carried out again to obtain the new natural frequency and it was found to be $34.92 \mathrm{~Hz}$ in the $80 \%$ case and $34.85 \mathrm{~Hz}$ in the $50 \%$ case.

Finally, nonlinear transient analyses were carried out at the new natural frequencies to find the energy dissipation characteristics.

The behavior of the joints in the $80 \%$ case and the $50 \%$ case are shown in Figure 13 and 14 
respectively. The stress and strain of one element in each joint unit is shown. As discussed above, the other element in the unit has almost the same behavior. The stiffness of the elements were different as it was assumed the joint stiffness was proportional to the macro-slip force. The stress-strain data was converted to force and displacement by use of the dimensions of the joints (cubes of $0.05 \mathrm{~m}$ on each side). Then the energy dissipated in the joint, which was proportional to the area of the loop was calculated. The loop size of Element 1 is smallest. Those of Element 2-4 are intermediate and the loop size of Element 5 is somewhat larger than those of Element 2-4. The loop sizes of Element 6-9 are largest. This indicates the different energy dissipating capabilities of joint groups. The energy dissipated in all these joints was summed and it was found to be $11.8 \mathrm{~J}$ in the $80 \%$ case and $28.2 \mathrm{~J}$ in the $50 \%$ case. The input energy was also calculated from the force-displacement at the excitation points on the base of satellite. In the $80 \%$ case the input energy was $67.1 \mathrm{~J}$ and in the $50 \%$ case it was $69.2 \mathrm{~J}$. Thus, in the $80 \%$ case the energy dissipated in the bolted joints was $17 \%$ of the input energy and in the $50 \%$ case it was $41 \%$.

The amount of macro-slip in each joint unit in the $50 \%$ case is shown in Figure 15 . The yield stress is shown in Figure 16. It can be seen that the joint's macro-slip increased with the yield stress in the joint, but was also constrained by the geometry. For example, joint unit 1 has almost no macro-slip because there was a small force transferred at this position and it was close to a 'rigid' edge that constrained the movement. The macro-slip in joint unit 4 was smaller than joint unit 2 and 3 because this joint was close to the middle point where the excitation point was and the panels were connected rigidly to transfer the large excitation force. In general, the top edges had more macro-slip than the bottom ones because they transferred more shear force and they were less constrained. The amount of macro-slip in the joints varied significantly, but the 
maximum macro-slip in all these joints was never over $0.15 \mathrm{~mm}$. This does not exceed the clearance between bolts and holes of the joints, which are normally $0.5 \mathrm{~mm}$.

It can be seen that the joints on the top of the satellite play an important role in dissipating energy. The case where only these joints go into macro-slip was considered. It was found that the natural frequency of the satellite was $34.91 \mathrm{~Hz}$ and the energy dissipated in the joints was $18 \%$ of the total energy input to the satellite.

\section{Comparison of responses of satellite systems with joints operating in the micro-slip and macro-slip regions.}

The satellite model incorporating bolted joints operating in micro-slip was almost the same as shown in Figure 8, except that all the elasto-plastic solid elements were removed and the two nodes on the panels were constrained by MPC forces to represent the micro-slip joints.

In order to see how the macro-slip force affects the response of the satellite, one node and one element on the spacecraft, (node $\mathrm{M}$ and element $\mathrm{N}$ in Figure 8) were chosen. The variation of the displacement of the node with the percentage of reduced yield stress is shown in Figure 17. The variation of the von Mises stress of element $\mathrm{N}$ is shown in Figure 18.

It can be see that when the macro-slip force decreased (reduced yield stress) the response of the spacecraft decreased too. The rate of change of displacement increased with reducing macro-slip force. The same was true for the stress. From the micro-slip condition to the $50 \%$ case the displacement decreased by about $29 \%$ and the stress decreased by about $26 \%$. This has considerable significance for a spacecraft. For example, a stress decrease means the weight of the 
spacecraft may be decreased because less material (or different materials) can be used to provide the necessary strength. This will decrease the whole cost of the spacecraft and the launch.

A more common way to represent the damping capability is through the damping ratio $\varsigma$. Figure 19 shows the frequency responses of two systems near their first natural frequencies. The damping ratio $\varsigma$ was obtained from the results using the half power bandwidth approach.

For the micro-slip joint model the damping was only from the structural damping. Since the energy dissipated in the micro-slip joints was very small it was reasonable to ignore their effect. Frequency response analysis was carried out on the model and the value of the damping ratio $\varsigma$ was found to be $2 \%$. This is entirely reasonable as the structural damping coefficient is double the damping ratio.

For the macro-slip data the effect of the joints should not be neglected because the energy dissipated in the joints is a significant part of the total energy. A series of non-linear transient analyses were carried out for the $50 \%$ case. These data are plotted in Figure 19. The damping ratio $\varsigma$ was found to be $3.5 \%$. Therefore, the damping ratio has increased significantly by incorporating macro-slip in the joints

\section{Conclusions}

In this paper a novel way of estimating the energy dissipated in bolted joints operating in macroslip in a spacecraft structure was introduced. It was used in a simple satellite model to assess the effect of macro-slip. Two important conclusions were obtained:

- By using proper parameters, the elasto-plastic solid element pair was able to represent the 
macro-slip response of bolted joint in a large structure accurately and efficiently.

- By using macro-slip joints the modal damping ratio can increase significantly, thus the force and displacement response of a spacecraft can be decreased and this provides an efficient way to decrease vibration of a spacecraft.

In fact this novel unit of two elasto-plastic solid elements can be used in structures of any complexity to represent the macro-slip behavior of bolted joints where, in general, it is not possible to include the detailed FE joint models in the global model.

\section{Notation:}

$\begin{array}{lll}\mathrm{A} & = & \text { area of shear surface; } \\ \mathrm{E} & = & \text { elastic modulus; } \\ \mathrm{E}^{\mathrm{T}} & = & \text { slop of stress-strain curve; } \\ F_{y} & = & \text { yield force; } \\ \mathrm{G} & = & \text { shear modulus; } \\ \mathrm{K} & = & \text { stiffness; } \\ \mathrm{L} & = & \text { element edge length; } \\ v & = & \text { Poisson's ratio; } \\ \sigma_{v m} & = & \text { von Mises stress; } \\ \sigma_{x}, \sigma_{y}, \sigma_{z} & = & \text { normal stresses; } \\ \sigma_{y l d} & = & \text { yield stress; and } \\ \tau, \tau_{y z}, \tau_{z x}, \tau_{x y} & = & \text { shear stresses; }\end{array}$




\section{References}

Crocombe A. D., Wang R., Richardson G., Underwood C. I. (2006). "Estimating the Energy Dissipated in a Bolted Spacecraft at Resonance", Computers and Structures, 48(5-6), 340-350.

Esmailzadeh E., Chorashi M., Ohadi A. R. (1996). “Analysis of Preloaded Bolted Joints under Exponentially Decaying Pressure", Journal of Pressure Vessel Technology (Transactions of the ASME) (USA), 118(4), 393-398.

Esmailzadeh E., and Chorashi M. (1996). “Optimal Design of Pre-Loaded Joints under Dynamic Loadings”, Proceedings of the International Conference on Pressure Vessel Technology, ICPVT, 2, 7-13.

Gantes, C. J., and Lemonis, M. E. (2003). "Influence of equivalent bolt length in finite element modeling of Tstub steel connections", Computers and Structures, 81, 595-604.

Gaul L., and Lenz J., Sachau D. (1997). “Active Damping of Space Structures by Contact Pressure Control in Joints", Proceedings of the 15th International Modal Analysis Conference, 202-208.

Gaul L., and Lenz J. (1997). "Nonlinear Dynamics of Structures Assembled by Bolted Joints", J., Acta Mechanica, 125(1-4), 169-181.

Gaul L., and Nitsche R. (2001). “The Role of Friction in Mechanical Joints”, Applied Mechanics Review, 54 (2), 93-106.

Hitchings D. (1992). A Finite Element Dynamics Primer, NAFEMS.

Kess HR, Rosnow NJ, Sidle BC (2002). "Effects of Bearing Surfaces on Lap Joint Energy Dissipation", Proceedings of IMAC-XX: A Conference on Structural Dynamics, 603-610.

Krzyzanowski J., Skoczynski W., Augul K. (1999). "Mechanism of vibration energy dissipation in flange bolted 
joints”, Proceedings of the International Seminar on Modal Analysis, CONF 23; Vol 2, 1071-1076.

Lenz J., and Gaul L. (1995). "The influence of microslip on the dynamic behaviour of bolted joints", Proceedings of the 13th International Modal Analysis Conference, Vols 1 and 2 Proceedings of the Society of Photo-Optical Instrumentation Engineers (SPIE) 2460, 248-254.

Sun W. (1989). "Bolted joint analysis using ansys superelements and gap elements", 1989 Ansys Conference Proceedings, Vol 2 - Engineering Design Options : From Pcs To Parallels, 66-75.

Ungkurapinan N., Chandrakeerthy S. R. De S., Rajapakse R. K. N. D., Yue S. B. (2003). "Joint slip in steel electric transmission towers", Engineering Structures, 25, 779-788.

Wang R., Crocombe A. D., Richardson G., Underwood C. I. (2005). "Modelling of small satellite structures incorporating bolted joints with non-linear viscoelastic layer", 19th Annual AIAA/USU Conference on Small Satellites, Logan, USA, SSC05-IX-7. 
Table. 1. The yield stress and shear modulus used in the different solid elements

\begin{tabular}{ccccc}
\hline \multirow{2}{*}{$\begin{array}{c}\text { Element } \\
\text { ID }\end{array}$} & \multicolumn{2}{c}{$80 \%$ case } & \multicolumn{2}{c}{$50 \%$ case } \\
\cline { 2 - 5 }$(\mathrm{MPa})$ & $G(G P a)$ & $\sigma_{\text {yld }}(\mathrm{MPa})$ & $G(\mathrm{GPa})$ \\
\hline 1 & 1.43 & 1.86 & 0.892 & 0.613 \\
2 & 2.02 & 3.25 & 1.26 & 1.48 \\
3 & 2.39 & 4.11 & 1.49 & 2.02 \\
4 & 2.50 & 4.36 & 1.56 & 2.18 \\
5 & 1.80 & 2.73 & 1.12 & 1.16 \\
6 & 2.46 & 4.27 & 1.54 & 2.11 \\
7 & 2.78 & 5.02 & 1.74 & 2.59 \\
8 & 2.92 & 5.36 & 1.83 & 2.80 \\
9 & 2.96 & 5.45 & 1.85 & 2.85 \\
\hline
\end{tabular}


Figure 1 - A single solid element model, appropriately constrained

Figure 2 - Force-displacement response at a top node of the single element model

Figure 3 - Dynamic test data of an aluminum bolted double lap joint

Figure 4 - In-plane joint configuration

Figure 5 - Corner joint configuration

Figure 6 - Test setup for the in-plane joint

Figure 7 - Three fastener corner joint response under different preloads

Figure 8 - Model of satellite with the bolted joint units in place

Figure 9 - A single bolted joint unit benchmark model

Figure 10 - Experimental force-displacement results of a 3-fastener corner joint with superimposed bilinear response

Figure 11 - Comparison of experimental and numerical results of the single joint model

Figure 12 - The von Mises stress $(\mathrm{Pa})$ of the simple satellite model from a frequency response analysis with $2 \mathrm{~g}$ y-excitation at a frequency of $34.97 \mathrm{~Hz}$

Figure 13 -The stress-strain response of the joint elements when the slip stress is $80 \%$ of the rigidly bolted stress

Figure 14 -The variation of stress with strain of solid elements representing the joints when the slip stress is $50 \%$ of the rigidly bolted stress

Figure 15 - The macro-slip of each joint unit

Figure 16 - The yield stress of each joint unit

Figure 17 - The variation of the displacement of node $\mathrm{M}$ with different macro-slip forces

Figure 18 - The variation of the von Mises stress of element $\mathrm{N}$ with different macro-slip forces

Figure 19 - Frequency response at node $\mathrm{M}$ in the satellite with different joints 


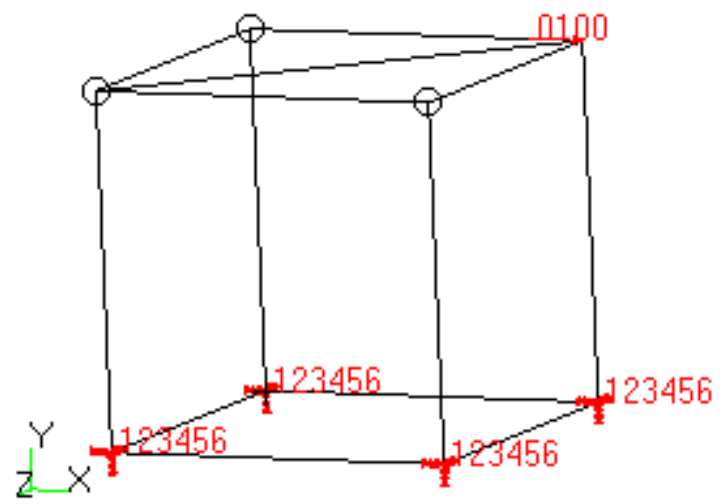

Figure 1 - A single solid element model, appropriately constrained 


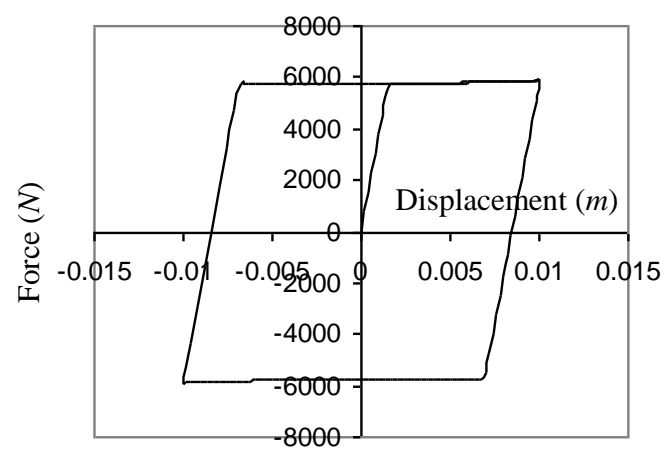

Figure 2 - Force-displacement response at a top node of the single element model 


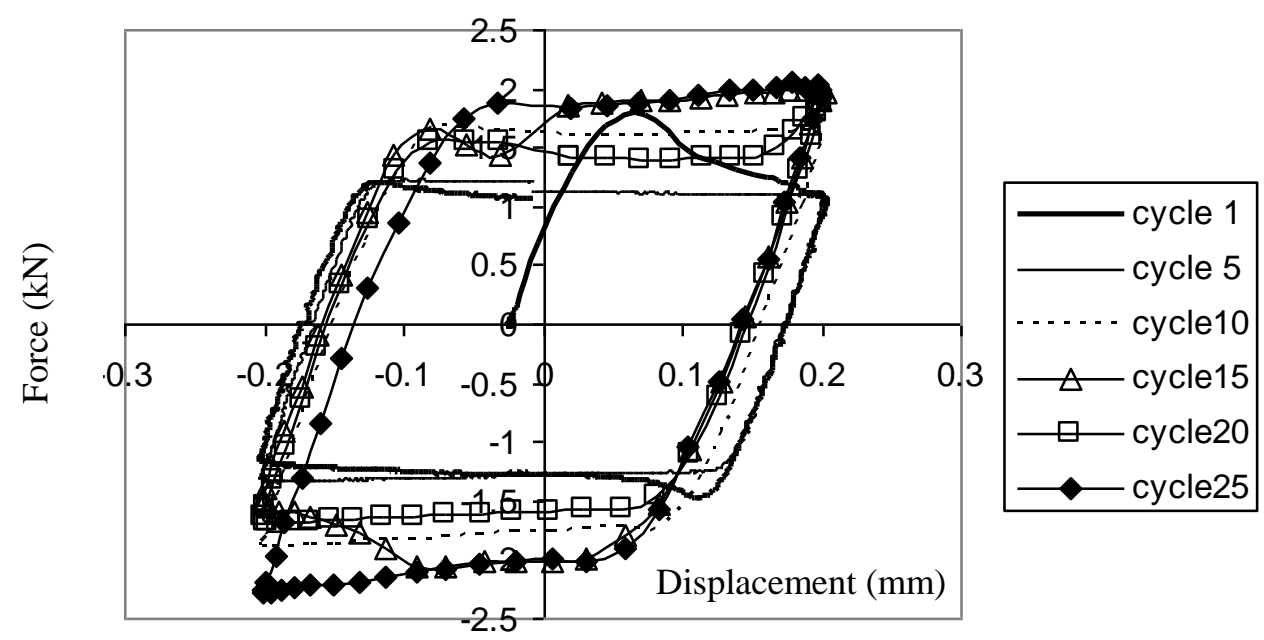

Figure 3 - Dynamic test data of an aluminum bolted double lap joint 

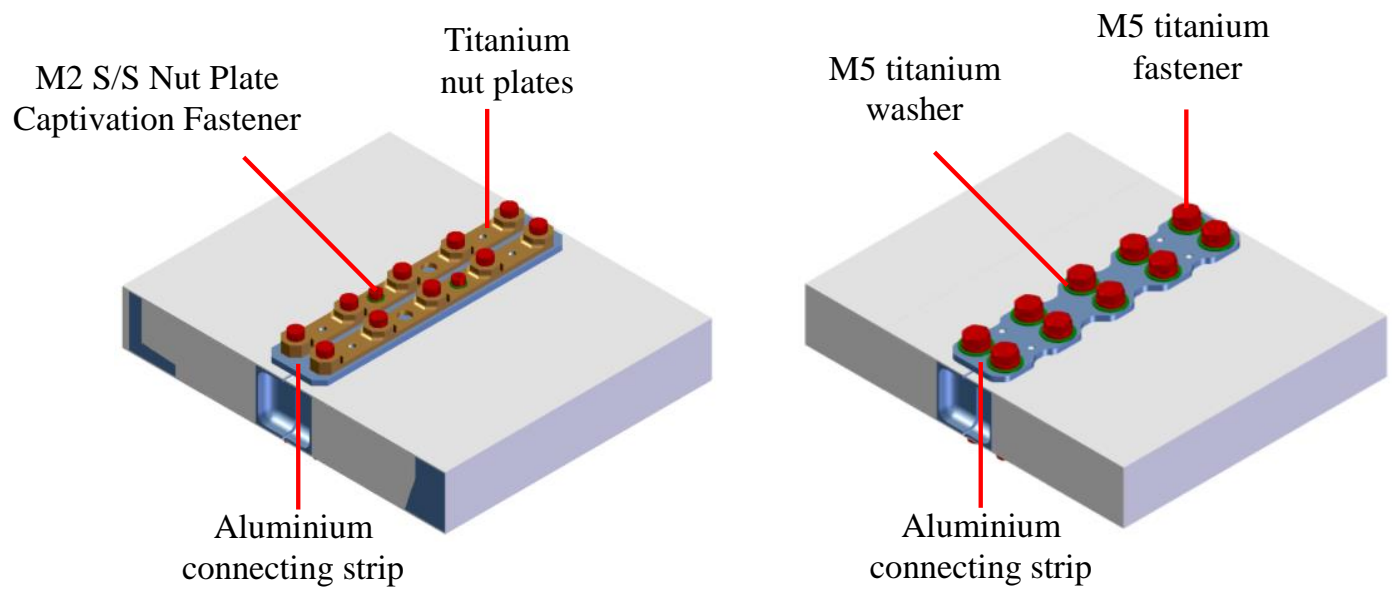

Figure 4 - In-plane joint configuration 

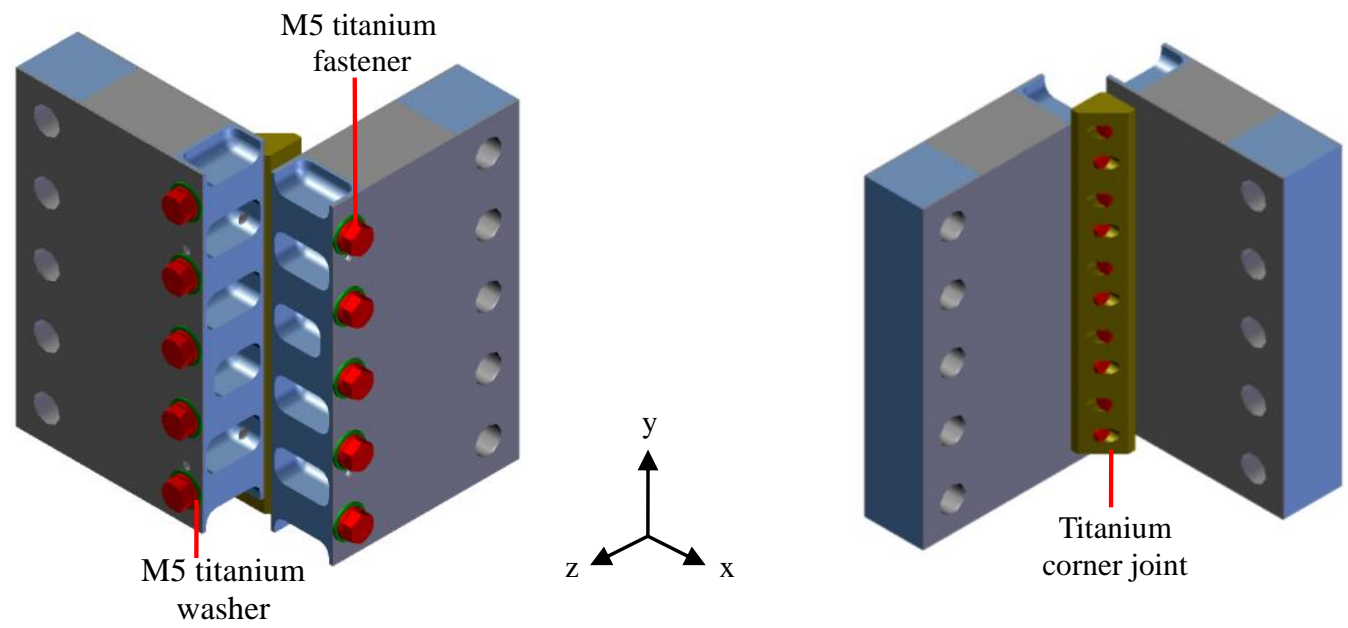

Figure 5 - Corner joint configuration 


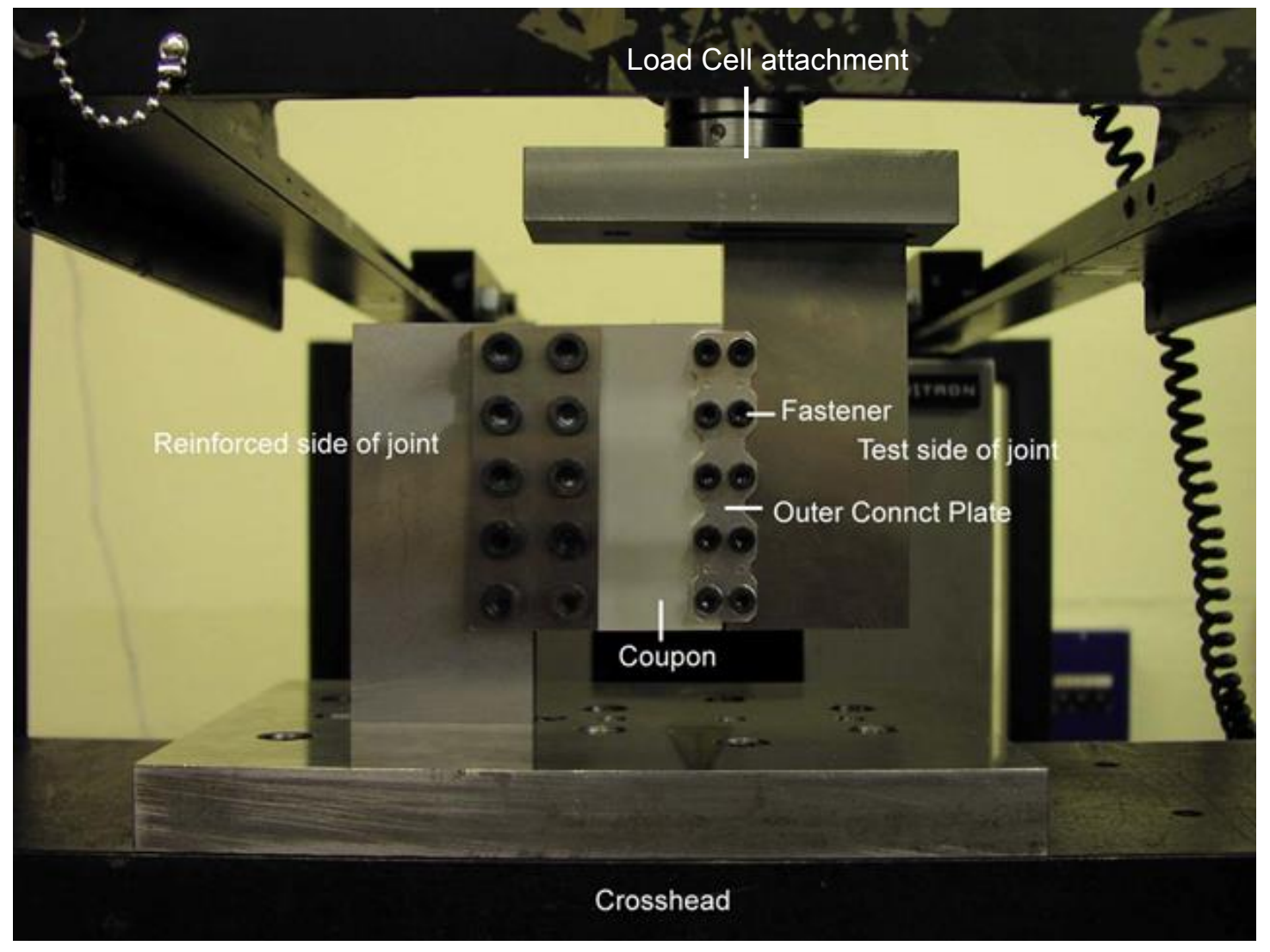

Figure 6 - Test setup for the in-plane joint 


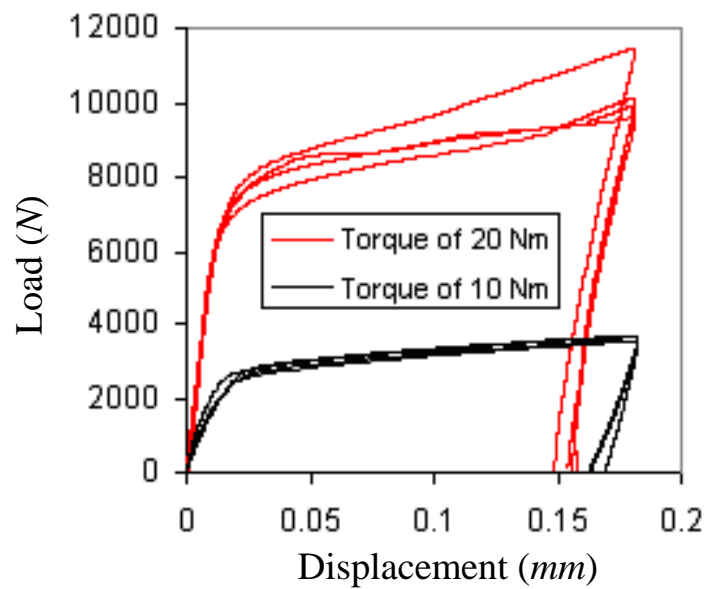

Figure 7 - Three fastener corner joint response under different preloads 


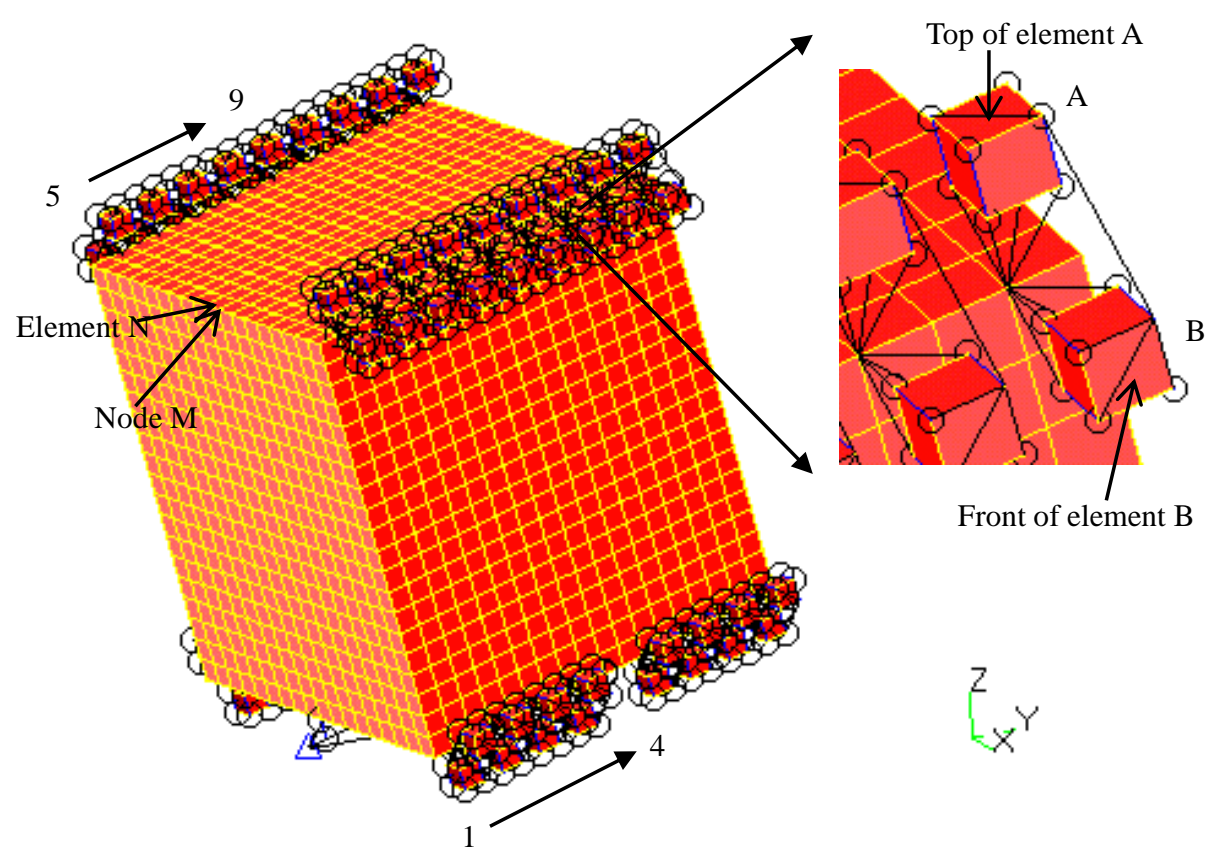

Figure 8-Model of satellite with the bolted joint units in place 


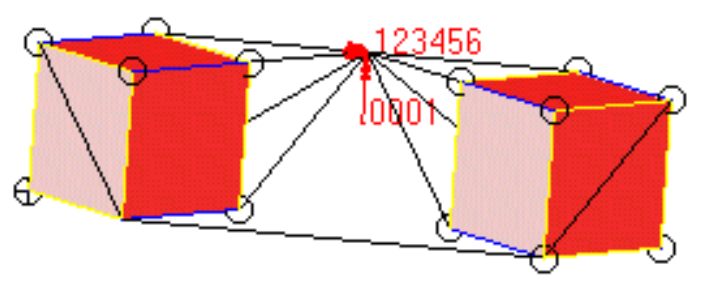

$L_{x}^{2}$

Figure 9 - A single bolted joint unit benchmark model 


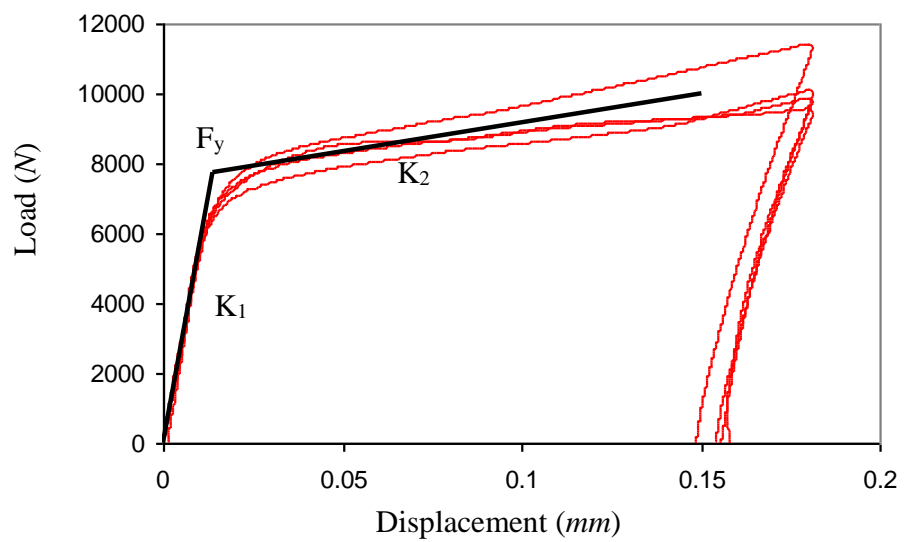

Figure 10 - Experimental force-displacement results of a 3-fastener corner joint with superimposed bi-linear response 


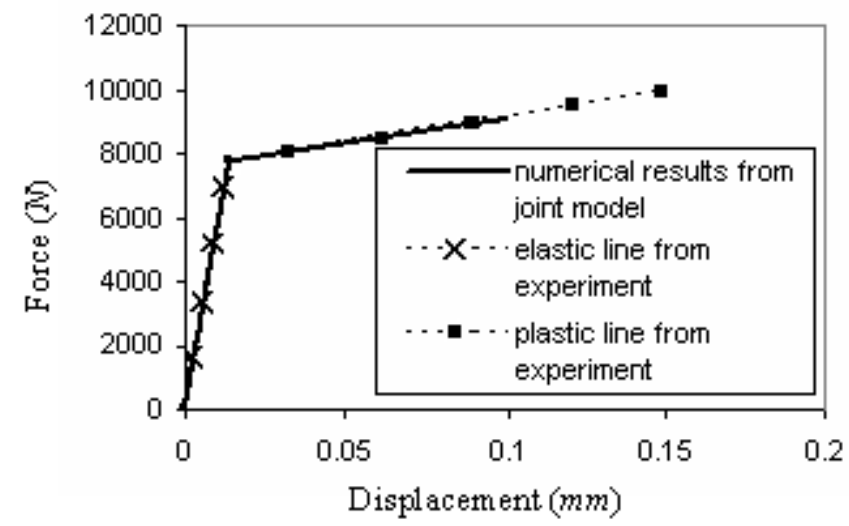

Figure 11 - Comparison of experimental and numerical results of the single joint model 

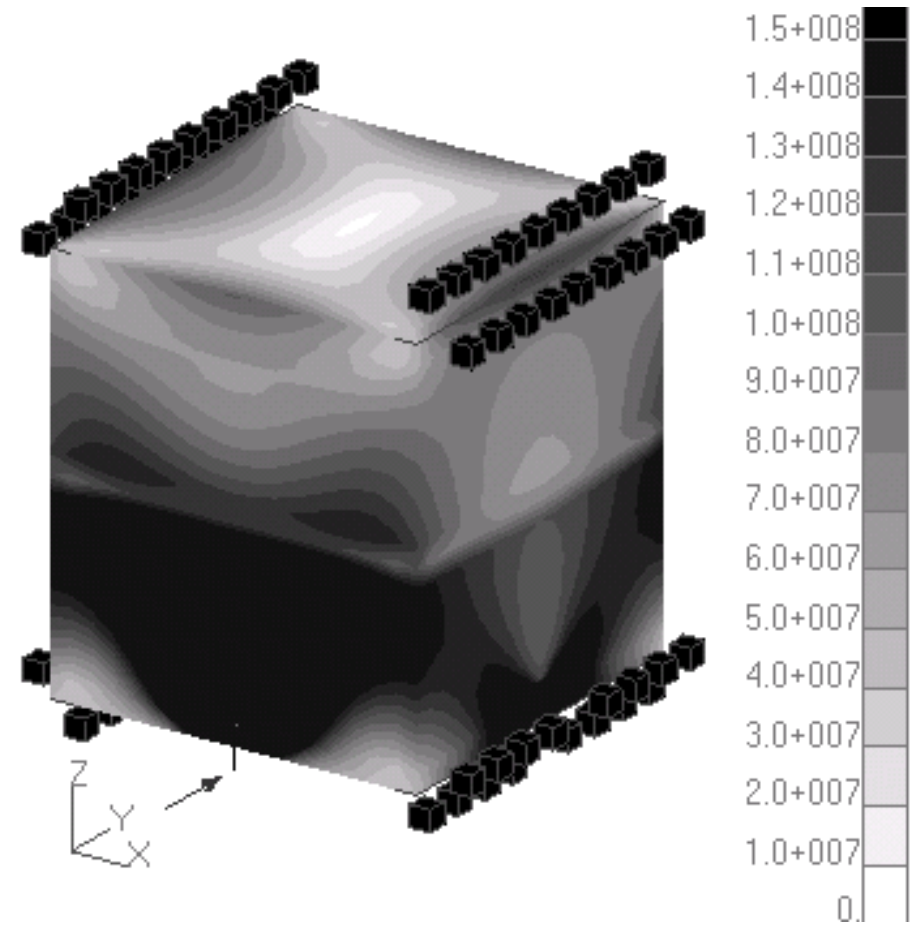

Figure 12 - The von Mises stress $(\mathrm{Pa})$ of the simple satellite model from a frequency response analysis with $2 \mathrm{~g}$ y-excitation at a frequency of $34.97 \mathrm{~Hz}$ 

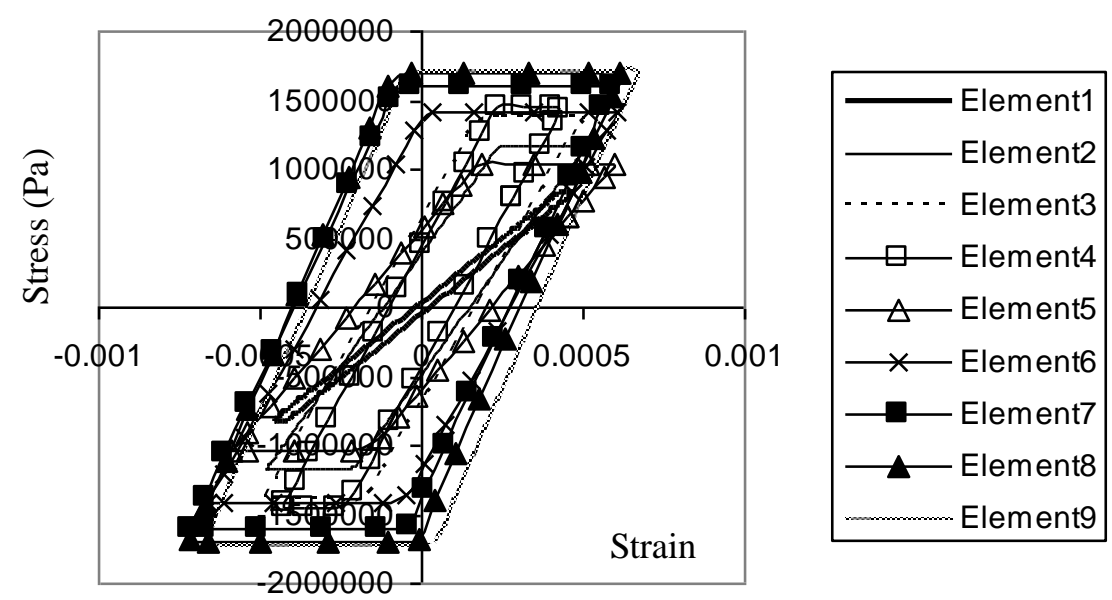

Figure 13 -The stress-strain response of the joint elements when the slip stress is $80 \%$ of the rigidly bolted stress 

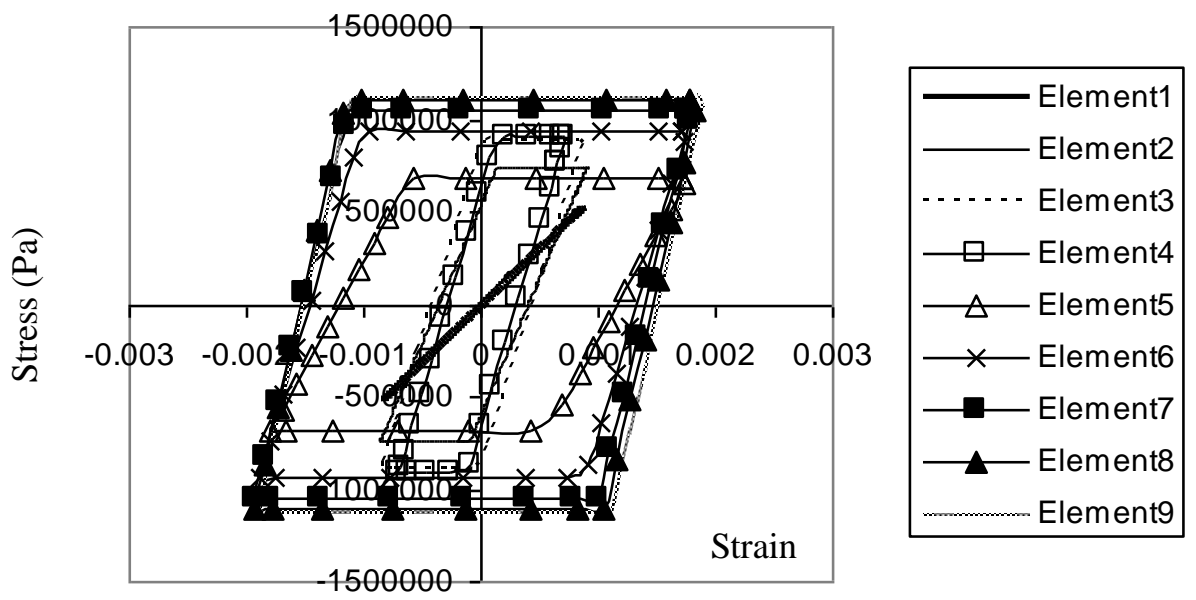

Figure 14 -The variation of stress with strain of solid elements representing the joints when the slip stress is $\mathbf{5 0 \%}$ of the rigidly bolted stress 

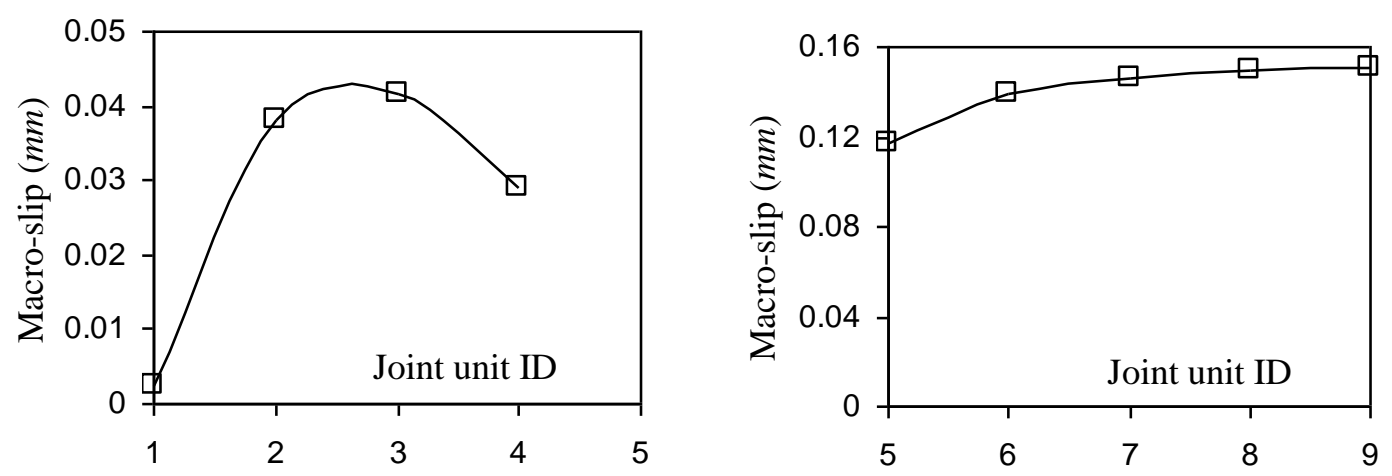

Figure 15 - The macro-slip of each joint unit 

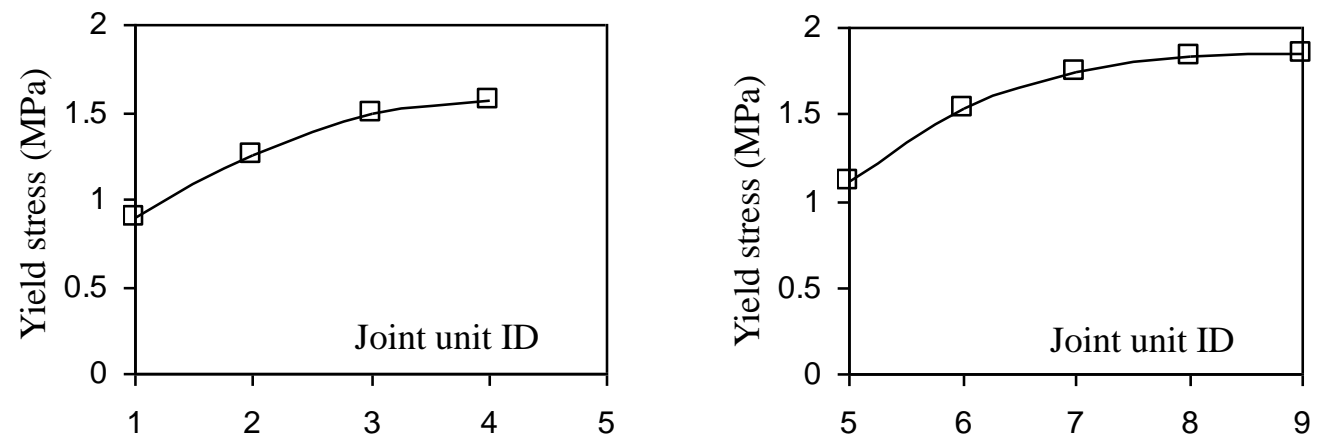

Figure 16 - The yield stress of each joint unit 


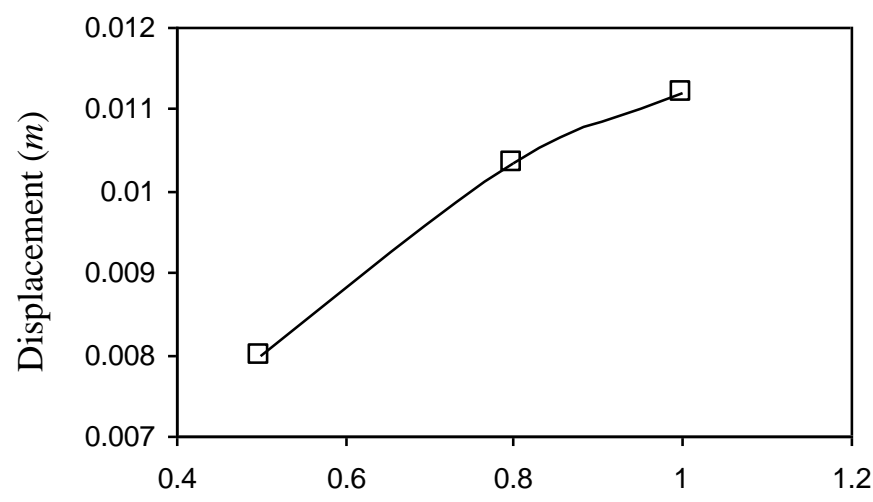

The ratıo of reduced yıeld stress to orıginal stress (no macro-slip)

Figure 17 - The variation of the displacement of node $M$ with different macro-slip forces 


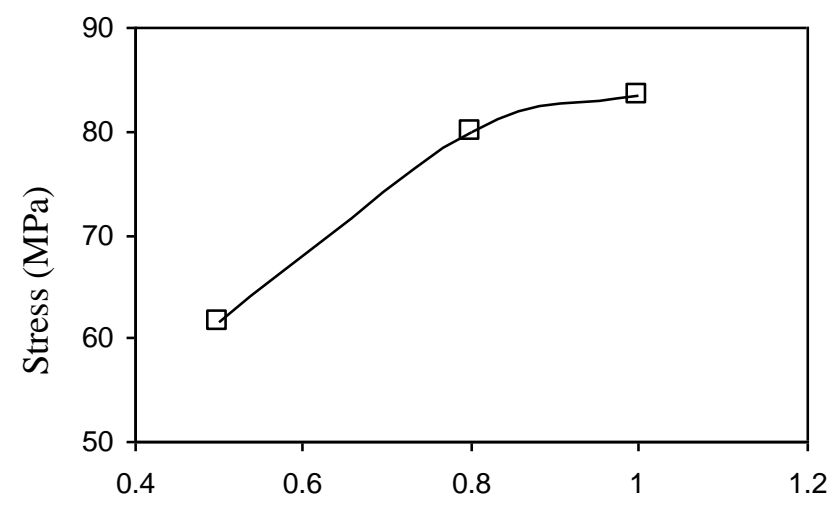

The ratio of reduced yield stress to original stress (no macro-slip)

Figure 18 - The variation of the von Mises stress of element $\mathbf{N}$ with different macro-slip forces 


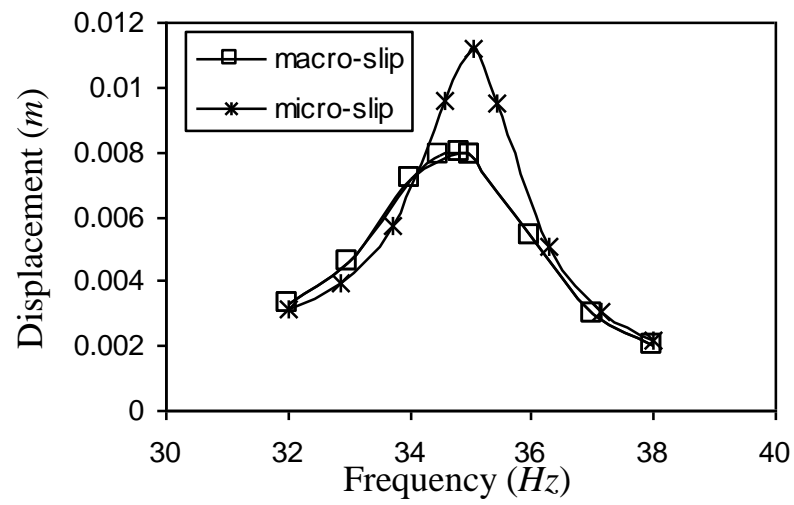

Figure 19 - Frequency response at node $M$ in the satellite with different joints 